\title{
Actuation Mechanisms of Carbon Nanotube-based Architectures
}

\author{
Sebastian Geier ${ }^{1}$, Thorsten Mahrholz ${ }^{1}$, Peter Wierach ${ }^{1}$, Michael Sinapius ${ }^{2}$; \\ ${ }^{1}$ Institute of Composite Structures and Adaptive Systems, \\ German Aerospace Center (DLR), \\ Lilienthalplatz 7, Braunschweig, Germany \\ ${ }^{2}$ Institute of Adaptronics and Function Integration, \\ Technical University of Braunschweig, \\ Langer Kamp 8, Braunschweig, Germany
}

\begin{abstract}
State of the art smart materials such as piezo ceramics or electroactive polymers cannot feature both, mechanical stiffness and high active strain. Moreover, properties like low density, high mechanical stiffness and high strain at the same time driven by low energy play an increasingly important role for their future application. Carbon nanotubes (CNT), show this behavior. Their active behavior was observed 1999 the first time using paper-like mats made of CNT. Therefore the CNT-papers are electrical charged within an electrolyte thus forming a doublelayer. The measured deflection of CNT material is based on the interaction between the charged high surface area formed by carbon nanotubes and ions provided by the electrolyte. Although CNT-papers have been extensively analyzed as well at the macro-scale as nano-scale there is still no generally accepted theory for the actuation mechanism. This paper focuses on investigations of the actuation mechanisms of CNT-papers in comparison to vertically aligned CNT-arrays. One reason of divergent results found in literature might be attributed to different types of CNT samples. While CNT-papers represent architectures of short CNTs which need to bridge each other to form the dimensions of the sample, the continuous CNTs of the array feature a length of almost $3 \mathrm{~mm}$, along which the experiments are carried out. Both sample types are tested within an actuated tensile test set-up under different conditions. While the CNT-papers are tested in water-based electrolytes with comparably small redox-windows the hydrophobic CNT-arrays are tested in ionic liquids with comparatively larger redox-ranges. Furthermore an in-situ micro tensile test within an SEM is carried out to prove the optimized orientation of the MWCNTs as result of external load. It was found that the performance of CNT-papers strongly depends on the test conditions. However, the CNT-arrays are almost unaffected by the conditions showing active response at negative and positive voltages. A micro alignment as result of tensile stress can be proven. A comparison of both results point out that the actuation mechanism strongly depends on the weakest bonds of the architectures: Van-der-Waals-bonds vs. covalent C-bonds.
\end{abstract}

Keywords: carbon nanotube, actuator, Bucky-paper, CNT-array, liquid electrolyte, solid electrolyte, actuation mechanism

\section{INTRODUCTION}

The discovery of carbon nanotubes was first published in $1952^{1}$ and again in $1974^{2}$ but the rolled up graphitic sheets finally found great scientific attention in $1991^{3}$ after Iijima's publication. Extensive research revealed early excellent properties such as high stiffness of $1 \mathrm{TPa},{ }^{4}$ strength of $41 \mathrm{GPa}^{4,5}$ and high thermal conductivity of $6000 \mathrm{~W} / \mathrm{Km}^{6}{ }^{6}$ These characteristics seem to open up an almost endless number of possible future applications. But the transfer of theoretical knowledge into commercially available applications turns out to be extremely challenging due to cheap mass-production and quality problems of the produced nanotubes ${ }^{7}$ which causes a performance gap between nano-scale and macroscopic structures. Furthermore, the rise of graphene since 2004 results in dwindling scientific interest. However, CNTs still attract interest in fields of nanoscale engineering

Further Author Information: Send Correspondence to Sebastian Geier

E-mail: Sebastian.Geier@dlr.de, Telephone: +495312952355 
such as nano-scale transistors for future super-fast computers ${ }^{8}$ or almost friction-less nano-scale motors ${ }^{9}$ and new properties are again and again discovered such as the remote joule heating. ${ }^{10}$

Baughman $^{11}$ was the first who revealed also their active behavior. Using mats made of randomly oriented carbon nanotubes he generated with his set-up free strain up to $0.2 \%$. This publication set the standard for the experimental set-up of CNT-mats: two electrodes in capacitor mode, one working electrode and its counter electrode, both made of CNT-papers. These sheets are immersed in an electrically insulating but ionic conductive electrolyte such as salty water. Furthermore the three electrode cell is completed by a Calomel reference electrode. The analyzed paper is activated via rectangular charge-pulses (maximum free strain) or triangular voltage (cyclovoltammetry) and shows an almost quadratic correlation between positive deflections and voltage steps, independent of the polarity. Considering the electromechanical properties of this carbon material, it seems to be a promising structure-integrated electromechanical transducer. Furthermore it overcomes most of the disadvantages other scientific relevant active materials like piezo ceramics (PZT), shape memory alloys (SMA), shape memory polymers (PMA) or electroactive polymers (EAP) suffer from. Currently piezo ceramics are the most application-oriented smart materials. The active mechanism, also known as the inverse piezo effect, can be understood as a crystalline deformation within an electric field generating high actuation frequencies up to $60 \mathrm{kHz}^{12}$ at a stiffness of $64 \mathrm{GPa}^{12}$. In contrast their inherent brittleness, low active strain $\left(0.15 \%{ }^{12}\right)$ and high operation voltage up to $200 \mathrm{~V}$ to $2800 \mathrm{~V}^{13}$ as well as their high density are very often severe drawbacks. Higher active strains of $8 \%^{12}$ can be generated by shape-memory alloys (see SMA in Figure 1) using a thermal or force-induced change of their crystalline metallic configuration from austenite into martensite. Slow reaction, an unstable long term behavior and hysteresis effects are thier adverse characteristics. The actuation-mechanism of the third group, the electro-active polymers abbreviated as EAP (see Figure 1), is based on volume change by ion diffusion or magnetic-induced electrostatic attraction. Thus, free outstanding free strains of $215 \% 14$, frequencies of $82 \mathrm{~Hz}^{14}$ (depending on mechanism) and low material densities are promising properties but their low stiffness does not allow highly loaded structural applications. In Figure 1 an overview of the most common active materials regarding their mechanical and active properties is given. Furthermore the mechanical and active properties of state of the art CNT-papers and CNT-yarns ${ }^{15}$ are also illustrated to point out the potential of this material. As an outlook, the ideal properties of CNTs are presented for better comparison.

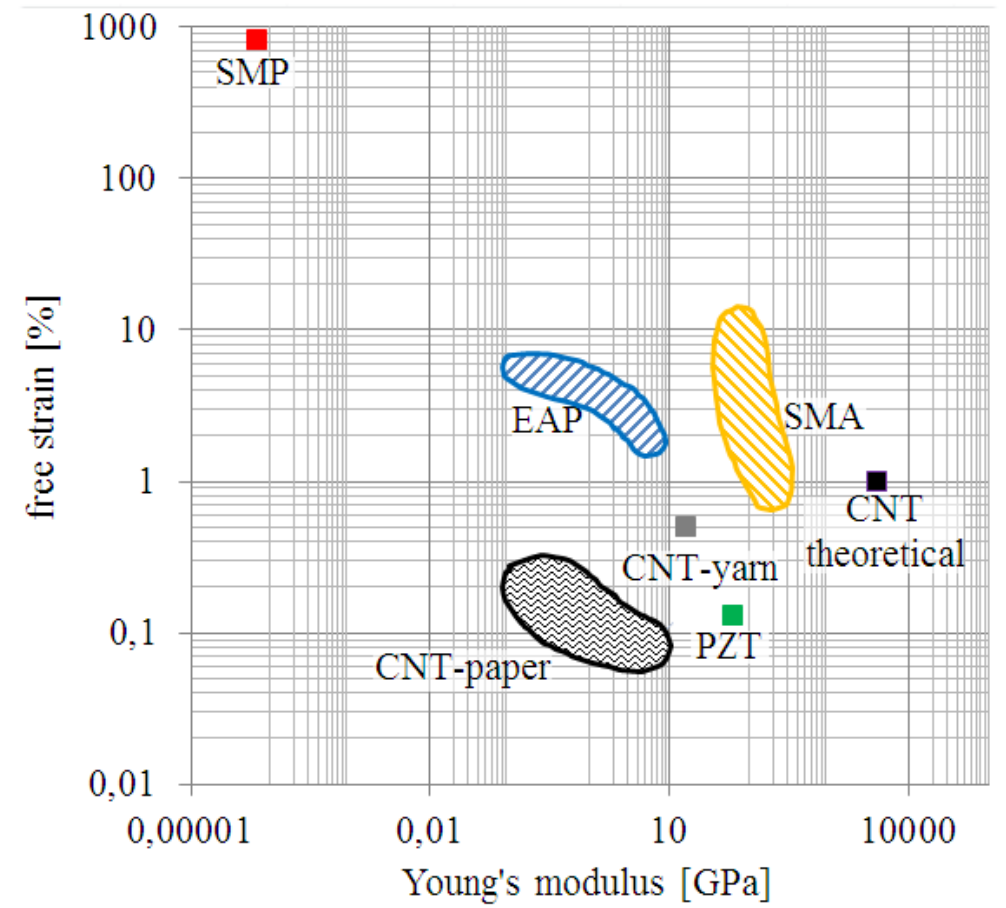

Figure 1. Performance of the most common smart materials ${ }^{12}$ PZT, EAP and SMA in comparison to CNT state of the art and its further potential as yarn or ideal actuator based on theoretical calculations or nano-scale experiments. 
However, published results like free strain or actuation force of CNT-based actuators have to be treated with circumspection because the results can either depend on the composition of the analyzed CNT-based material or on the used analysis method or secondary effects or combination of the mentioned effects. In this context the term architectures will be used in this paper to represent structures of macroscale dimensions which are formed by individual CNTs. Usually the length of the individual single tubes is too small to form one dimension of a macroscopic architecture. Furthermore the reactive surface of single-walled nanotubes tend to reduce the energy by clustering in bundles. A CNT-bundle represents the first step towards an architecture. The carbon tubes within an architecture are entangled with each other or linked by van der Waals forces. Thus results found by testing CNT-based architectures can only represent partially the active behavior of the single tubes because the linking represents the weakest part of the system. Using out of-plane set-ups to analyze CNT-papers in bending mode $^{11}$ give also rather qualitatively results because secondary effects like thermal- or diffusion-induced volume change can influence the findings extensively. For reducing these effects, the measurements are performed in the pre-stressed mode at the in-plane test set-up. ${ }^{16}$ In this case the composition of the tested material has to be considered as symmetrical or homogeneous. Various macro-scale tests cannot give a satisfactory explanation for the effect as well as nanoscale analyses like atomic force microscopy of single tubes ${ }^{17}$ or Raman-spectroscopy of CNT-papers. ${ }^{18}$ Suppiger $^{19}$ supposed an electrostatic mechanism because of an inverse correlation between Young's modulus and free strain of CNT-papers. Whitten ${ }^{20}$ revealed strong dependency between the mechanical properties of a paper and the test condition, whether it is tested in dry or wet state. Furthermore the use of different types of electrolytes, aqueous electrolytes vs. ionic liquids, indicates an influence of the ions because the Young's modulus is reduced significantly by the large, ionic, charged molecules. In addition Spinks ${ }^{21}$ explained the deflections of CNT-papers by their composition similar to honeycombs, which acting like a pneumatic actuator driven by gas generation. Thus, CNT-papers seem to be rather influenced at their tube linking instead of transferring a reaction of the CNT-structure. This paper focuses on collecting and presenting indications which mechanism might be the reason for of CNT-paper actuation.

As alternative material arrays of multi-walled carbon tubes are tested which already reach microscale dimensions regarding their length. This characteristic makes them usable for testing. Yun ${ }^{22}$ used similar materials, named towers of CNTs and measured their active performance. The weak mechanical linking between tubes and the silicon substrate as well as the curly shape of the individual tubes and used high voltage steps $\pm 2 \mathrm{~V}$ beyond the redox-window of aqueous electrolytes $( \pm 1 \mathrm{~V})$ reduced the significance of the results. In further publications different types of MWCNT-arrays ${ }^{23}$ are investigated to find correlations between the active behavior and the tube-morphology, degree of orientation and -length.

The presented paper focuses on randomly oriented single-walled nanotube-papers, also called bucky-papers, to reveal their condition- and morphology-dependencies. In contrast aligned multi-walled carbon nanotube (MWCNTs)-arrays are tested under the same conditions. The results will be compared and help to evaluate the significance of the specific material to identify the mechanism behind the carbon nanotube actuation. Furthermore the micro tensile test of the MWCNT-arrays will be presented in order to prove the ability of a macro-scale facility to align micro/nano-scale material.

\section{EXPERIMENTAL}

The experimental chapter is divided into four parts. Fristly the quality assessment and sample preparation is presented. In the second section the test set-up of the actuated tensile tests will be explained. Furthermore, in the third section the micro tensile test set-up is shown. Finally the used formulae will be presented.

\subsection{Quality assessment of used material and sample preparation}

All used materials such as the supplied CNT-powder, the manufactured papers and the MWCNT-arrays are selectively analyzed using scanning electron microscopy (LEO 1550, Zeiss Jena AG, working distance of 7 mm, operating voltage of $5 \mathrm{kV}$ ). Ideas about the composition of the material and how to handle it are in particular derived from the morphology of the CNT-agglomerates and the evidence of the type of CNTs (single or bundled single-walled CNTs vs. MWCNTs). The upper and lower surfaces of the sheets are analyzed by SEM but because of pressure-induced compaction it is difficult to detect detail of the tubes. A better insight can be achieved by analyzing the cross section. In order to avoid an unintended surface as result of cutting the cross section has to be prepared by a cryo-cracking. 
The basis material of CNT-papers is CNT-powder which is supplied by Thomas Swan Ltd. (Elicarb 0925, Thomas Swan Ltd.) containing single- and double-walled carbon nanotubes of d diameter range of $1 \mathrm{~nm}$ to 3 $\mathrm{nm}$ and a purity of $95 \%$. Due to the formed bundles an accurate mean length of the individual tubes cannot be provided. The CNT-paper is produced using a pressure assisted filtration process. At first an aqueous solution containing $99 \mathrm{~g}$ deionized water, one gram surfactant (sodium dodecyl sulfate, SDS, with a purity of $99 \%$ supplied by Sigma-Aldrich Co. LCC.) and $0.1 \mathrm{~g}$ SWCNT-powder is prepared. This solution is homogenized in an ultrasonic bath at maximum power (Sonorex Digital 10P, Bandelin Electronic GmbH \& Co. KG) for 180 minutes. The prepared suspension is filtered using a pressure of 6 bar. The SWCNTs are deposited on a polycarbonate (PC) membrane with $400 \mathrm{~nm}$ pores (Track-Etche polycarbonate membrane 23006-47, supplied by Satorius AG). Then the sheet is rinsed with $600 \mathrm{ml}$ deionized water to remove the surfactant that may coat the CNTs which would electrically insulate them. Finally the paper must be peeled off the membrane and is pressed between two PTFE-based blocks by $1.87 \mathrm{~Pa}$ in an oven at $80^{\circ} \mathrm{C}$ (UFP $500 \mathrm{M} 1 \mathrm{~T} 300 \mathrm{C}$, Memmert GmbH \& Co. $\mathrm{KG}$ ) for at least four hours. The paper is cut into samples with a mean length of $10 \mathrm{~mm}$, a width of $1.5 \mathrm{~mm}$ and a thickness of $0.14 \mathrm{~mm}$.

The CNT-arrays consist of vertical free standing, unaffected CNTs, typically grown on a silicon substrate. The MWCNT-arrays are supplied by the University of Hamburg-Harburg. The tubes are grown during 5.5 hours using a thermal-induced chemical vapor deposition method. Their geometry, also described in detail by Vainio, ${ }^{24}$ features a mean length of $2.8 \mathrm{~mm}$ (continuous tubes), tube diameters in the range of $17 \mathrm{~nm}$ to $37 \mathrm{~nm}$ with a varying angle $\alpha_{C N T}$ of $0^{\circ}$ to $43^{\circ}$ as result of an overall sinusoidal shape. The wavy shape might be a result of the low density of the array. Typically CNTs support each other during the growth which is not as effective when they are not standing in high density. The specimens are manufactured from a complete cross-section of the array which is cut off using a sharp razor blade. The as-produced specimen thickness is about $1 \mathrm{~mm}$. In a subsequent step the cross-section is divided into a set of specimens with almost the same width. These samples must be carefully removed from the razor blade using tweezers. To compress the sample in order to increase its bulk-density it is positioned between two microscope slides. The manual compression already achieves a 80-97 \% reduction of the thickness $(11 \mu \mathrm{m})$. Further compacting can be conducted using a manual hydraulic press (Atlas manual 25 tons hydraulic press GS25011, Specac Limited, UK) by applying a load of 5 tons which implies an average pressure of $5450 \mathrm{MPa}$. The final geometry of the samples is a length of $2.8 \mathrm{~mm}$, a width of $2 \mathrm{~mm}$ and a thickness of $10 \mu \mathrm{m}$.

A digital microscope (VHX-1000, Keyence Corporation) is used to measure the exact length and width of the sample geometries. The thickness is measured using a micrometer screw (IP 54, Mitutoyo Europe GmbH). To avoid structural damage the samples are positioned between two glass-slides during the thickness-measurement. The thickness of the slides is measured afterwards and subtracted from the original result.

\subsection{Actuated tensile test set-up}

A standard tensile testing device (Z005, Zwick GmbH \& Co. KG) is the basis of the actuated tensile test. The machine is supplemented by two clamping jaws and a cylindrical reservoir made of polytetraflurethylene (PTFE). The reservoir is filled up to the bottom of the upper mounting with electrolyte. The wetting ensures the ionic interconnection of the samples/working electrode, the reference electrode and the platinum counter electrode during the experiment. PTFE remains electrochemically inert during charging of the samples which avoids contamination as a result of chemical reactions between the corrosive, salty electrolyte and parts made of base materials such as metal or steel. It is well known that PTFE is a comparatively soft material and thus CNTs cannot be tested to their extremum. The aim of these experiments is a qualitative comparison of samples made of two different materials based on carbon tubes: randomly oriented single-walled nanotube-based architectures versus multi-walled carbon nanotubes of macroscopic length. A schematic picture of the cross section on the left and a detailed view of the test set-up on the right are shown in Figure 2. The tests are conducted at a speed of $0.03 \mathrm{~mm} / \mathrm{min}$ to avoid prior mechanical damage. To obtain sufficient resolution a $10 \mathrm{~N}$ load cell (KAP-Z, Zwick $\mathrm{GmbH} \& \mathrm{Co}$. KG) is used. Experiences of the accuracy of the two available testing modes (deflection- and load-controlled) delivered by the testing device result in the selection of deflection controlled tests. Consequently the results are recorded with the force as the dependent variable. The load- and deflection-data is recorded by the software TestExpert II V3.31, also provided by Zwick.

The CNT-samples are arranged similarly to an in-plane strain test set-up which can be considered as the 


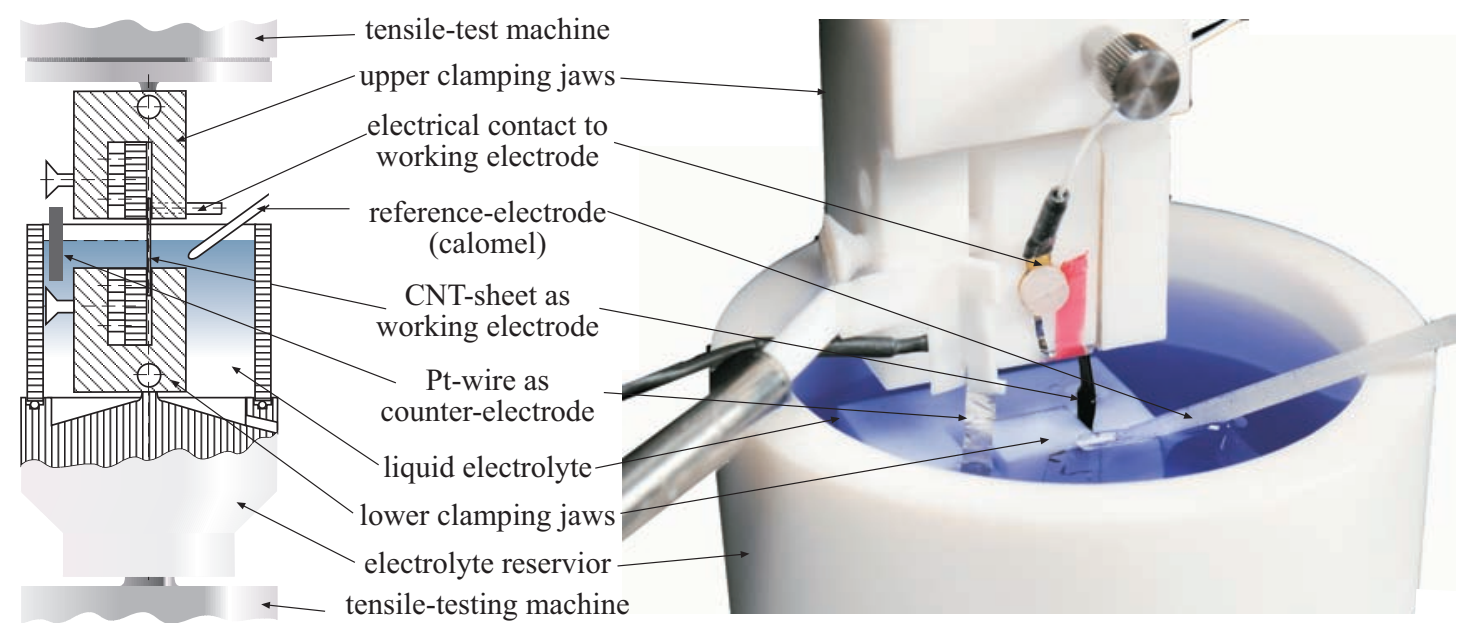

Figure 2. Overview of the tensile test set-up:

left: Schematic sketch of the cross section of the test set-up.

right: Detailed view onto the three electrode cell.

standard test set-up and is described elsewhere ${ }^{16}$. Within the three electrode cell the CNT-sheet represents the working electrode. For reasons of reproducibility a Pt-wire acts as counter electrode. A calomel electrode (KE 10, Sensortechnik Meinsberg GmbH) acts as the reference electrode. The working and the counter electrode are arranged like a capacitor with the reference electrode in between, positioned closer to the working electrode. All electrodes are immersed in a specimen-specific electrolyte. While the CNT-papers exhibit lower surface energy a water-based electrolyte, sodium nitrate $\left(\mathrm{NaNO}_{3}\right.$, Sigma-Aldrich Co. LCC) is used. As consequence of the high surface energy of the arrays resulting in extreme hydrophobicity only ionic liquids, in the case of the presented study, 1-ethyle-3-methylimidazolium bis(trifluormethyl-sulfonyl)imide (EMImTFSA, IoLiTec GmbH), can be used. The cell is controlled by a potentiostat (1030 PC.T., IPS Elektroniklabor GmbH \& Co. KG) and is charged via a function generator (FG 300, Yokogawa Deutschland $\mathrm{GmbH}$ ). The results are recorded via a data acquisition system (SCM05, LMS International). Both clamping jaws are equipped with electrodes for measuring the electrical resistivity of the sample to indicate damage to the specimen caused during mounting or inaccurate clamping.

Previously published tests are conducted under varying conditions until mechanical failure. The measurement of mechanical strength is critical for the use of brittle CNT-papers. However, testing until mechanical failure does not represent the design operating principle of a commercial actuator. For this reason only one specimen (per electrolyte) is tested within its elastic range. All deviations resulting from material inhomogeneities in the master-paper can be avoided. The relative differences between the different conditions are analyzed. The absolute results are only of secondary interest. In the presented test series the specimens are immersed in their individual electrolyte for at least 30 minutes so that they become saturated with ions and to avoid material swelling during the test. Furthermore as a result of slight shifting and deformation of the clamps during the installation of a sample, tensile or compression stresses can occur. Therefore the clamping length is individually adjusted in order to start all tests in unloaded condition. As an additional test the paper sample is investigated under preloaded condition of $0.2 \mathrm{~N}$. The arrays are also investigated under a pre-stressed condition $(0.015 \mathrm{~N})$ in order to reduce the waviness and improve their alignment. The specimens are charged constantly throughout the test using voltage steps of $\Delta \pm 0.5 \mathrm{~V}$ and $\Delta \pm 0.9 \mathrm{~V}$ according to their redox-window of $\pm 1 \mathrm{~V}$ and loaded up to an uncritical force of $0.03 \mathrm{~N}$ until the test is stopped. For the arrays the voltages steps are extended within the redox-window of EImTFSA of $\pm 2 \mathrm{~V}$. This procedure is repeated at least six times using the same voltage step. Afterwards the specimen is tested in uncharged condition to enable relaxation of the material. The campaign continues using higher voltage steps within the range of the redox-window for negative and positive voltages around the zero potential of $0.1 \mathrm{~V}$. The Young's moduli of the different steps are compared afterwards and are 
calculated between $0.04 \mathrm{~N}$ and $0.14 \mathrm{~N}$. In the case of preloaded conditions the calculation was carried out at $0.019 \mathrm{~N}$ and $0.029 \mathrm{~N}$. The free length between the clamping jaws is $5 \mathrm{~mm}$ for paper experiments and $0.5 \mathrm{~mm}$ to $1 \mathrm{~mm}$ for testing arrays.

\subsection{Micro tensile test set-up for testing MWCNT-arrays}

According to the actuated tensile testing the array samples are tested almost the same way but in dry condition and installed within the chamber of a SEM Leo 1550, Zeiss Jena AG) for in-situ observation of the tube alignment. The micro tensile test facility is individually manufactured and supported by the Institute of Material Science of the German Aerospace Center (DLR). The load cell is designed for loads up to $500 \mathrm{~N}$ so that a low resolution has to be expected. To avoid prior mechanical damage a testing speed of $1 \mu \mathrm{m} / \mathrm{s}$ is used. The test set-up and further details are presented in Figure 3.

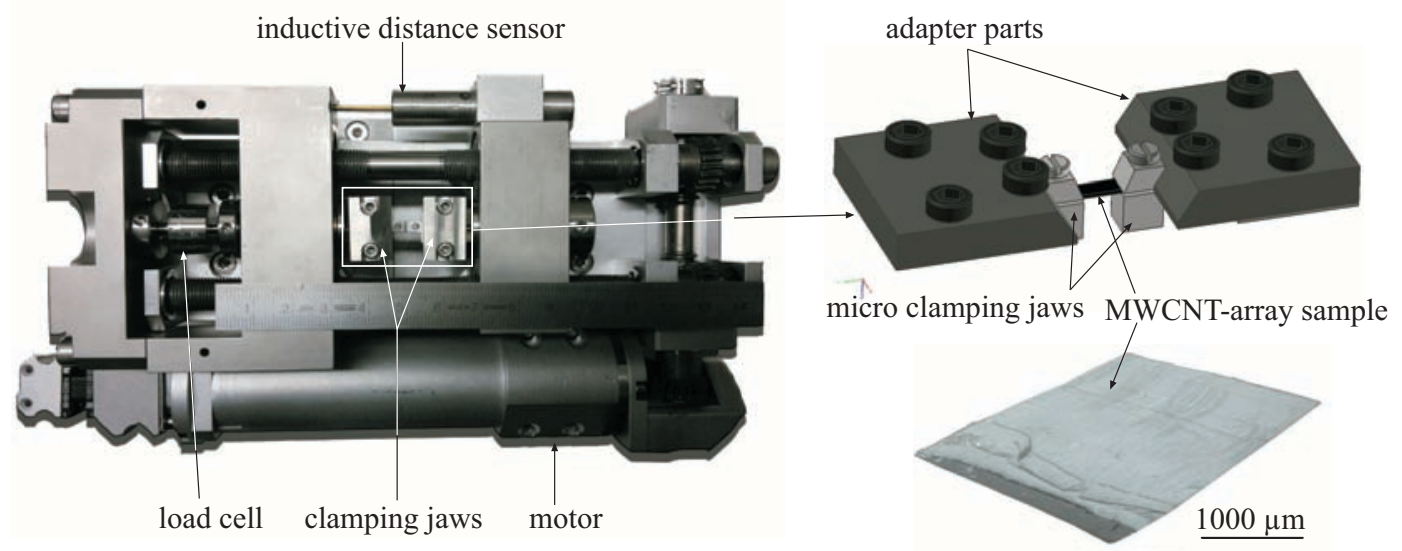

Figure 3. Overview of the micro tensile test set-up: left: Overview of the whole set-up which is adjusted on the positioning stage within the chamber of the SEM. right, on the top: Detailed view on the mounting. right, on the bottom: Detailed image of a positioned MWCNT-array sample.

It is expected that this test reveals the effect of applied macroscale forces on microscale architectures in terms of generating higher degrees of orientation. An improved orientation is needed for reducing effects such as swelling or electrostatic effects as result of the wave-induced misalignment. The SEM-images, using the secondary electron scattering, are recorded using an operating voltage of $5 \mathrm{kV}$ with a working distance of $18.4 \mathrm{~mm}$. The sample is loaded path controlled and the tests are divided by several holding steps for being able to take images of the whole sample and a detailed position which is observed during the whole tensile test. The sample is analyzed twice at every holding step. The first image of the array is taken with low resolution (200-times) for an overview of the sample condition whether it reveals structural damages or sliding traces. The second image is recorded with higher resolution (10000-times) to observe the micro/nanoscale shape, especially the alignment of the tubes.

\subsection{Used mathematical formulae}

The Young's modulus $\mathrm{E}$ of the material is calculated from the stress $\sigma_{n}$ using the measured force $\mathrm{F}_{n}$ and the specimen's cross section geometry caculated from $\mathrm{w}_{s p}$ and $\mathrm{t}_{s p}$ (compare equation $1^{25}$ ). As second parameter the relative strain $\varepsilon_{n}$, is calculated using the detected displacement $\Delta \mathrm{l}_{s p}$, see equation $2^{25}$. Finally $\mathrm{E}$ is calculated from equation 3 as the mean value of all points of the graph. At least five additional experiments with similar results should confirm that the experiments are only conducted in the linear-elastic region of the CNT-sheets. 


$$
\begin{gathered}
\sigma_{n}=\frac{F_{n}}{A_{s p}}=\frac{F_{n}}{w_{s p} \cdot t_{s p}}[\mathrm{MPa}] \\
\varepsilon_{n}=\frac{\Delta l_{s p}}{l_{0}}[\%] \\
E=\frac{\Delta \sigma}{\Delta \varepsilon}[\mathrm{MPa}]
\end{gathered}
$$

$\begin{array}{lll}A_{s p} & {\left[\mathrm{~m}^{2}\right]} & \text { specimen's cross-section area } \\ E & {[\mathrm{~Pa}]} & \text { Young's modulus } \\ \sigma_{n} & {[\mathrm{~Pa}]} & \text { mechanical stress } \\ \varepsilon_{n} & {[\%]} & \text { relative strain } \\ F_{n} & {[\mathrm{~N}]} & \text { force during test } \\ l_{0} & {[\mathrm{~m}]} & \text { free distance between clamp jaws } \\ w_{s p} & {[\mathrm{~m}]} & \text { width of sample } \\ t_{s p} & {[\mathrm{~m}]} & \text { thickness of sample }\end{array}$

\section{RESULTS AND DISCUSSION}

This section is divided into four subsections. The first part deals with the quality assessment of the manufactured samples to evaluate how the sample composition may influence the measured results. The second and third subsections present the results of the actuated tensile tests. Both types of specimen, the papers made of randomly oriented single-walled carbon nanotubes and the vertically aligned multi-walled carbon nanotube arrays, are discussed in their own section respectively. The fourth subsection reports the results of the micro-tensile tested MWCNT-arrays which are stretched in-situ within a scanning electron microscope to achieve a further alignment of the tubes.

\subsection{Quality assessment of CNT-paper and -array samples}

The SEM investigations of the CNT-papers are firstly presented, afterwards the findings of the MWCNT-arrays. While the lower and upper surfaces of CNT-papers reveal almost no details of the tube bundles as a result of the pressure-induced compacting the cross section view gives a detailed insight of the paper composition. The SEM images reveal a layer like build-up of flakes formed by agglomerated SWCNTs as it is shown in Figure 4 a). Due to less information about the composition it is not sure whether the layers are continuous or have rather small dimensions. During the dispersion process the agglomerates of the used powder are separated in individual tubes which are stabilized by the surfactant SDS. Taking the discussed images into account the dispersion process seems only to reduce the agglomerate size or the amount of tubes forming a bundle. During the filtration a twodimensional weight-induced recombination seems to take place to form the paper. Protruding bundles of CNTs bridge the gaps between the flakes, as Figure $4 \mathrm{~b}$ ) illustrates. The paper has to be considered as a continuous flake like structure, as Figure 4 c) depicts it, which can be used as a model structure for understanding the actuation mechanism of CNT-papers.

The curly shape of the arrays can be clearly observed in Figure 5 b) which is apparent along the whole length of the tubes (compare Figure 5 a)) but varies in its amplitude. Figure 5 c) reveals structural damages as result of pressure-induced compacting. Due to the local high stresses the array-tubes collapse so that a structural failure occurs nearby the mounting. Here great changes in stress occur within a small area so that the material, in this case the carbon bonds, are extremley stressed. Typically multi-walled tubes exhibit more structural defects which are too fragile to bear the stress.

\subsection{Results of the actuated tensile testing}

At first the results of the actuated SWCNT-paper tests are shown followed by the results of the tests conducted with MWCNT-arrays. 


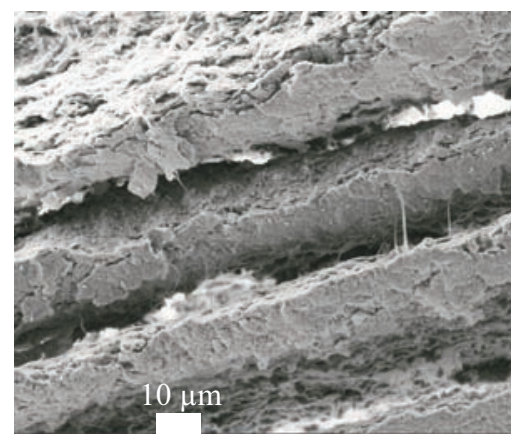

a)

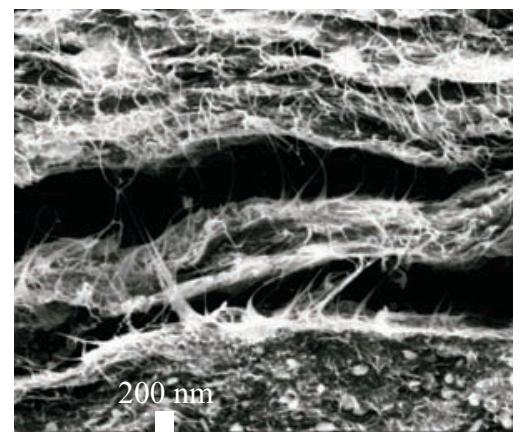

b)

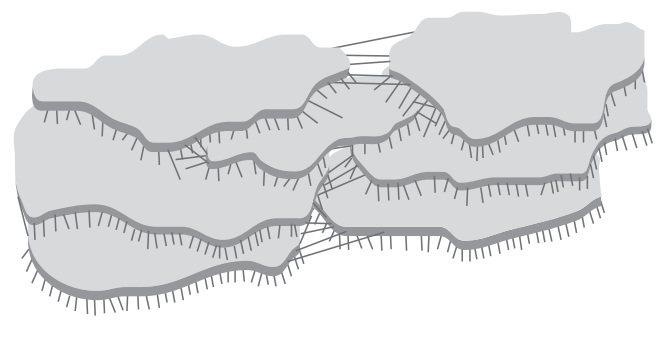

c)

Figure 4. SEM-images of SWCNT-papers:

a): Layer like build-up of a CNT-paper.

b): Bridging of SWCNT-bundles between layers of CNT-flakes.

c): Schematic illustration of a possible model structure representing the CNT-paper composition.

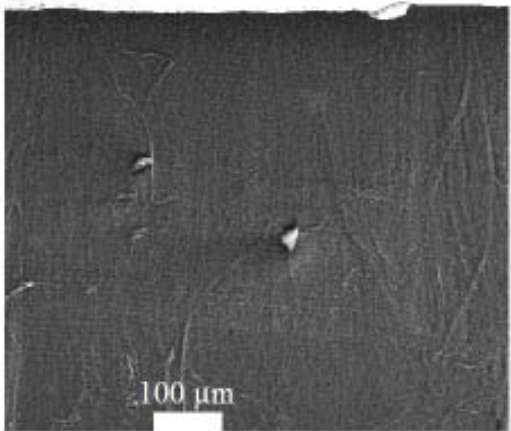

a)

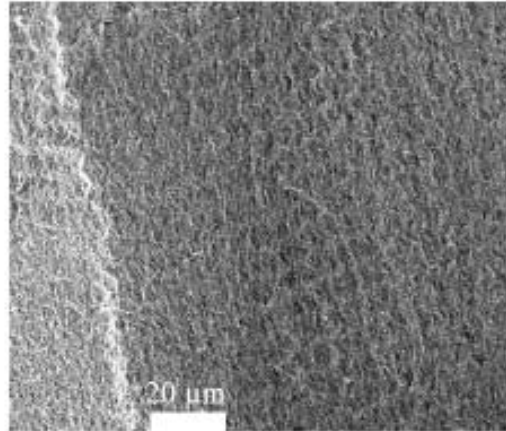

b)

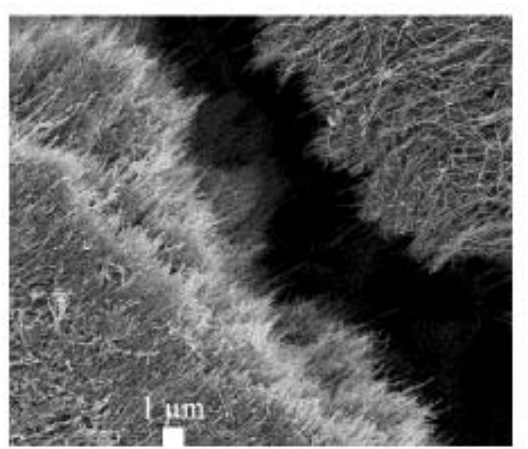

c)

Figure 5. SEM-images of MWCNT-arrays:

a): View on the upper surface of a prepared MWCNT-array specimen with low resolution revealing the waves of the curled CNTs.

b): Detailed view of the first image with higher resolution of the wavy tube shape.

c): View on the upper surface of a array-specimen showing the fracture line of the MWCNT-array specimen. 
Table 1. Comparison of the results using one molare sodium chloride until failure and testing within the elastic range

\begin{tabular}{cllll}
\hline volts & \multicolumn{2}{c}{ until failure } & \multicolumn{2}{c}{ elastic range } \\
& $\begin{array}{l}\text { mean } \\
\text { Young's } \\
\text { modulus }\end{array}$ & $\begin{array}{l}\text { standard } \\
\text { deviation }\end{array}$ & $\begin{array}{l}\text { Young's } \\
\text { modulus }\end{array}$ & $\begin{array}{l}\text { standard } \\
\text { deviation }\end{array}$ \\
& {$[\mathrm{MPa}]$} & {$[\%]$} & $5 \mathrm{MPa}]$ & {$[\%]$} \\
\hline $0 \mathrm{~V}$ & 198 & 33 & 529 & 0.3 \\
$-0.4 \mathrm{~V}$ & 228 & 16 & 531 & 0.7 \\
$-0.8 \mathrm{~V}$ & 270 & 8 & 515 & 1.4 \\
$+0.6 \mathrm{~V}$ & 152 & 60 & 508 & 1.1 \\
$+1 \mathrm{~V}$ & 147 & 69 & 276 & - \\
\hline
\end{tabular}

\subsubsection{Tensile tested SWCNT-papers under actuation}

In earlier tests ${ }^{26,27}$ the carbon papers were tested in actuated mode until mechanical failure. This approach turned out to be very material consuming and the results revealed large standard deviation which made it difficult to give certain statements about the observed effects. However, it was found that the sample quality varies although all sample were taken from the same master paper. Thus, in this paper the former approach was replaced by testing the same specimen within its elastic range (see Figure 6 a) and b)). This approach allows to carry out much more experiments and this test mode is similar to the real service condition of an actuator application. Table 1 compares the results of the former approach (third and fourth row) with the currently carried out tests in the elastic range of the papers.

Figure 6 b) demonstrates the reversible elastic behavior of a CNT-paper. As long as the paper is stressed with low forces of $0.03 \mathrm{~N}$ (comparative with the $0.8 \mathrm{MPa}$ ) the experiment can be repeated without a decreasing mechanical behavior. Furthermore the paper reveals high residual strength in Figure 6 a) so that it can be concluded that the paper is not damaged by the elastic tests.
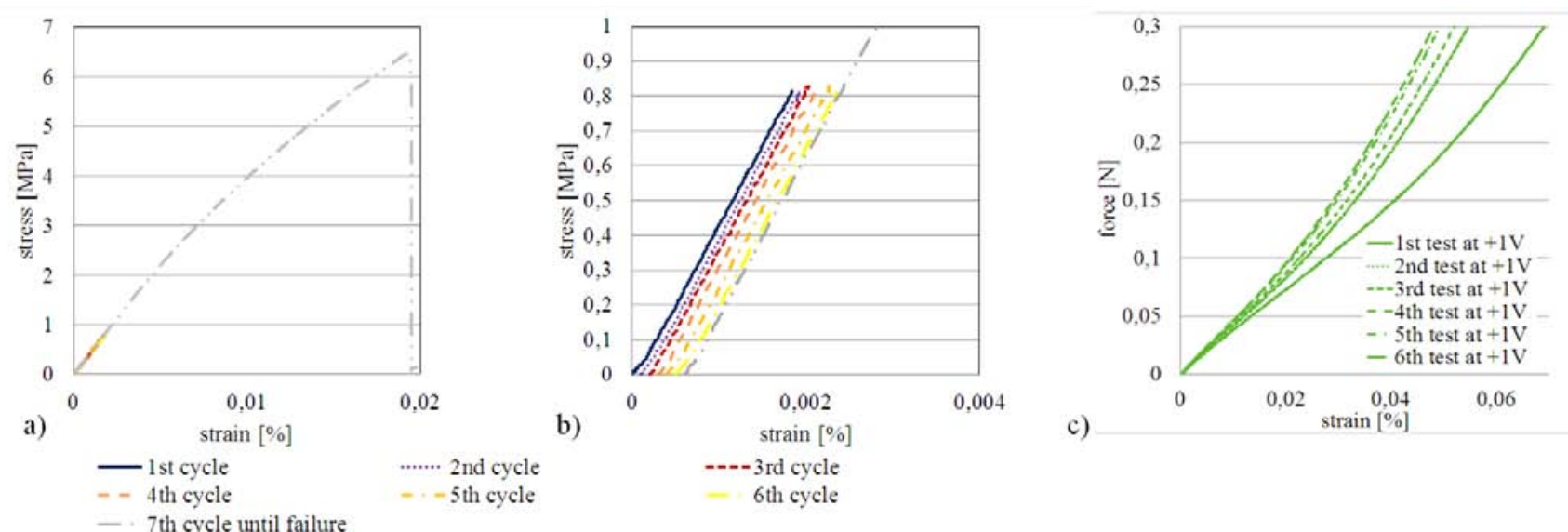

Figure 6. Results of tensile tested SWCNT-papers:

a): Overview of seven tensile tests within the elastic range of a paper $(0 \mathrm{~V})$.

b): Detailed view of the tensile tests within the elastic range and an added shift of $0.0001 \%$ for a better differentiation of the individual tests.

c): Relaxation process during a series of six tests with a charging of $+1 \mathrm{~V}$.

Table 1 reveals not only a strong dependency regarding the sample condition whether it is dry or wet, the recorded data also documents a loss of stiffness by charging. Furthermore, the level of voltage has also a significant 
influence. It can be found that the Young's modulus decreases by increasing the voltage. This strongly indicates an electrostatic reason for the reactions. In addition it turns out that positive charging generates lower losses compared to negative. This observed effect might be attributed to the different ion radii of the ions $\mathrm{Na}^{+}$and $\mathrm{NO}_{3}{ }^{-}\left(\mathrm{Na}^{+}\right.$crystalline: $95 \mathrm{pm}$, hydrated: $358 \mathrm{pm}$; $\mathrm{NO}_{3}{ }^{-}$crystalline: $264 \mathrm{pm}$, hydrated: $\left.335 \mathrm{pm}^{28}\right)$.

However, during the tests, especially during the charged series a relaxation of the Young's modulus can be observed (see Figure $6 \mathrm{c}$ ) at $+1 \mathrm{~V}$ ). While the Young's modulus of the first test is only 123 MPa, the Young's modulus recovers up to $152 \mathrm{MPa}$. Possible explanations are alignment processes within the paper. Another explanation is shown in Figure 7 a) to c)

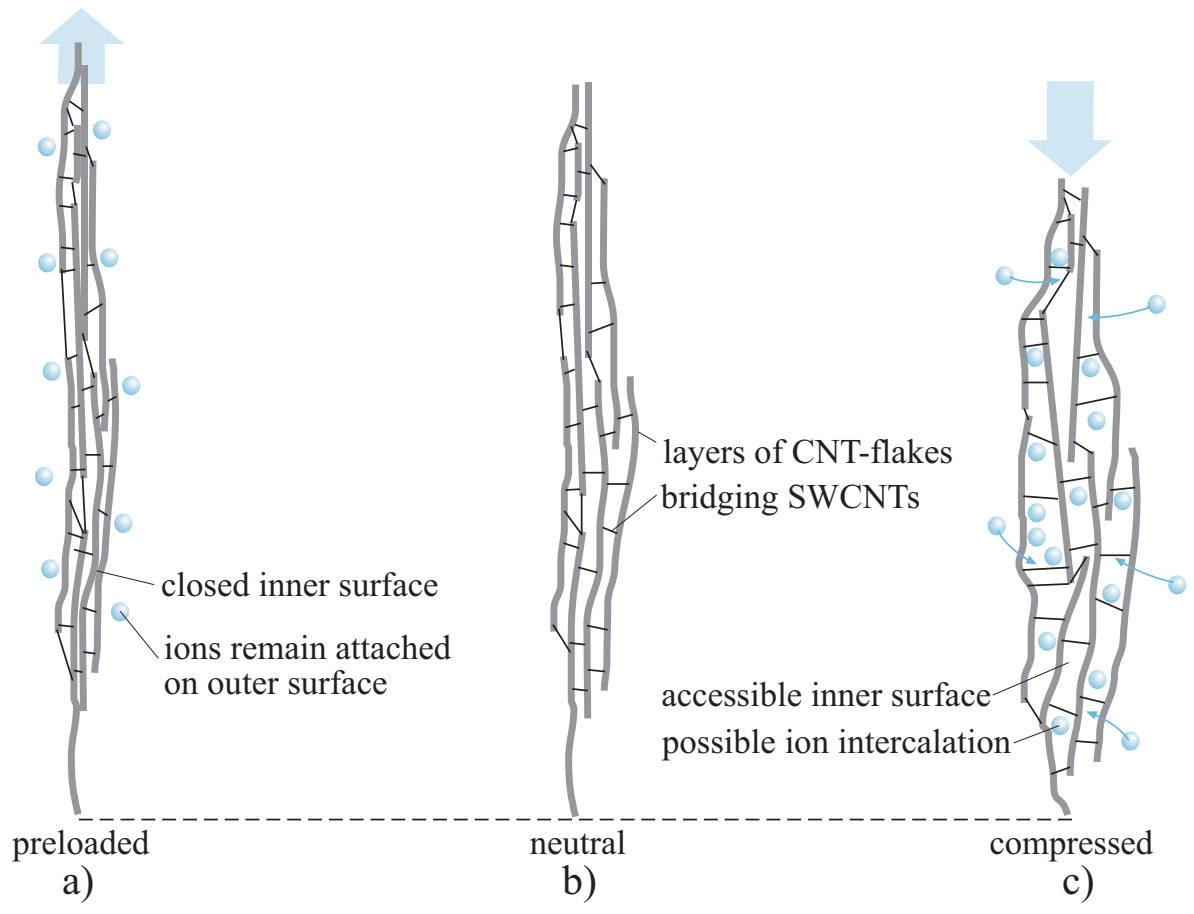

Figure 7. Force-induced conditions of a CNT-paper:

a): Preloaded condition of a paper with a closed composition and ions attached to the outer surface.

b): Neutral unloaded condition comparable to images of Figure 4.

c): Improved ion intercalation as result of compression.

In Figure $7 \mathrm{~b}$ ) the layer-like build-up of a CNT-paper is shown as it is found during SEM-analyses with the difference of having flakes instead of continuous layers. These flakes are interconnected by single carbon nanotubes. By compressing the paper, as illustrated in Figure $7 \mathrm{c}$ ), the gaps between the flakes open up for the diffusion of ions of the electrolyte which intercalate within the paper. The ions are softening the paper by forming an electrostatic double-layer around the flakes which cannot transfer forces. This ion layer acts like lubricants between the flakes, enabling an easier sliding of the flakes. As a result of higher charging more ions intercalate into the paper structure which causes decreasing Young's moduli. Furthermore, this case represents the first experiment within a series when the ions have enough time to fill up the structure. During a test series with every load-cycle ions are be removed out of the gaps what causes the increasing mechanical stiffness. By using a pre-load, as it is illustrated in Figure 7 a), the gaps of the paper remain closed and fewer ions are able to diffuse into the paper. The results are higher Young's moduli and a reduced relaxation. So this paper model easily explains all observed electromechanical properties of the paper at actuated tensile testing and at in-plain strain measurements. 


\subsubsection{Tensile tested MWCNT-arrays under actuation}

In Figure 8 a) it can be shown that even an array shows an elongation during charging. As result of the ionic liquid the redox-window is larger and a first effect can be detected at $1.3 \mathrm{~V}$. The tests are conducted until $\pm 2 \mathrm{~V}$ but due to the fact the cyclovoltammetry already reveals electrochemical reactions the found results should not be taken into account. In Figure $8 \mathrm{~b}$ ) the results of voltage, current, charge and force at voltage-steps of $-1.5 \mathrm{~V}$ and $+1.5 \mathrm{~V}$ are presented.
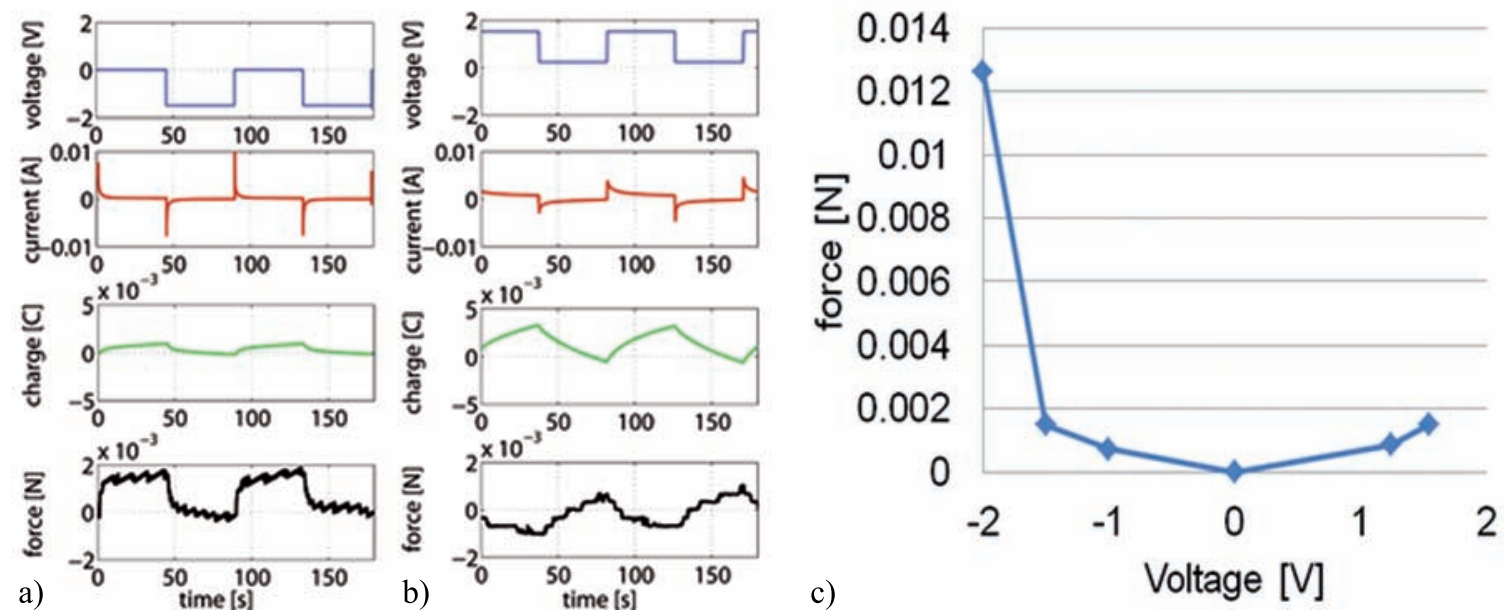

Figure 8. Results of the MWCNT-array actuation:

a): Voltage, current, charge and force-results recorded during actuation using -1.5 V.

b): Earlier mentioned data recorded during actuation using $+1.5 \mathrm{~V}$.

c): Actuation results found in the range of $\pm 2 \mathrm{~V}$.

The force results of both presented potentials are almost the same $(0.015 \mathrm{~N})$ but their shape are totally different. Another view on the charge may give the answer. While at $-1.5 \mathrm{~V}$ the force generation shows a stable behavior in charged mode the overall shape appears rectangular exhibiting sharp edges. In contrast the graphs at $+1.5 \mathrm{~V}$ show rather triangular shapes of the charge- and force-graphs. This indicates an ohmic share of the force generation, maybe also accompanied by irreversible chemical reactions pointing out the end of the electro-chemical window of the ionic liquid.

\subsection{Micro tensile tests of MWCNT-arrays}

The micro tensile test is conducted within a SEM. Therefore, no liquid electrolytes could be used. Even ionic liquids which are said to exhibit a high vapor pressure should not be tested (ILs can absorb water from the environment which would vaporize during evacuation within the SEM-chamber) to avoid serious contamination or even a damage of the SEM detectors. The influence of the electrolyte on the array-CNTs is not the focus of this test series. It is the aim to prove an alignment of the wavy MWCNTs and in what way the force- and deflection-data can be correlated with condition of the sample alignment. Figure $9 \mathrm{~d}$ ) presents the force-graph. As result of the low resolution of the load cell the force-graph exhibits a high deviation (blue graph). Using mean values (orange graph) reveals a clearer behavior of the arrays during the micro tensile test.

Within Figure 9 another five images of the test are presented which are taken at different phases and details of the test. The first pair of images (Figure 9 a)-1 and a)-2) show the whole specimen fixed between the mountings. In the second, detailed image the curly shape of the arrays can clearly be observed. The images a) -1 and a)-2 are taken at a certain position of the specimen before the test has been started. The second pair of images b)-1 and b)-2 documents the alignment at the same position. While in the image b)- 1 of the whole specimen deformed areas can be observed the detailed image b)-2 reveals an alignment of the former wavy MWCNTs. The images are taken at a deflection of $602 \mu \mathrm{m}$. At a deflection of $1521 \mu \mathrm{m}$ the specimen failed one the one hand by sliding out of the mounting (left section) and structural failure of the carbon tubes (right side of the specimen) as it 

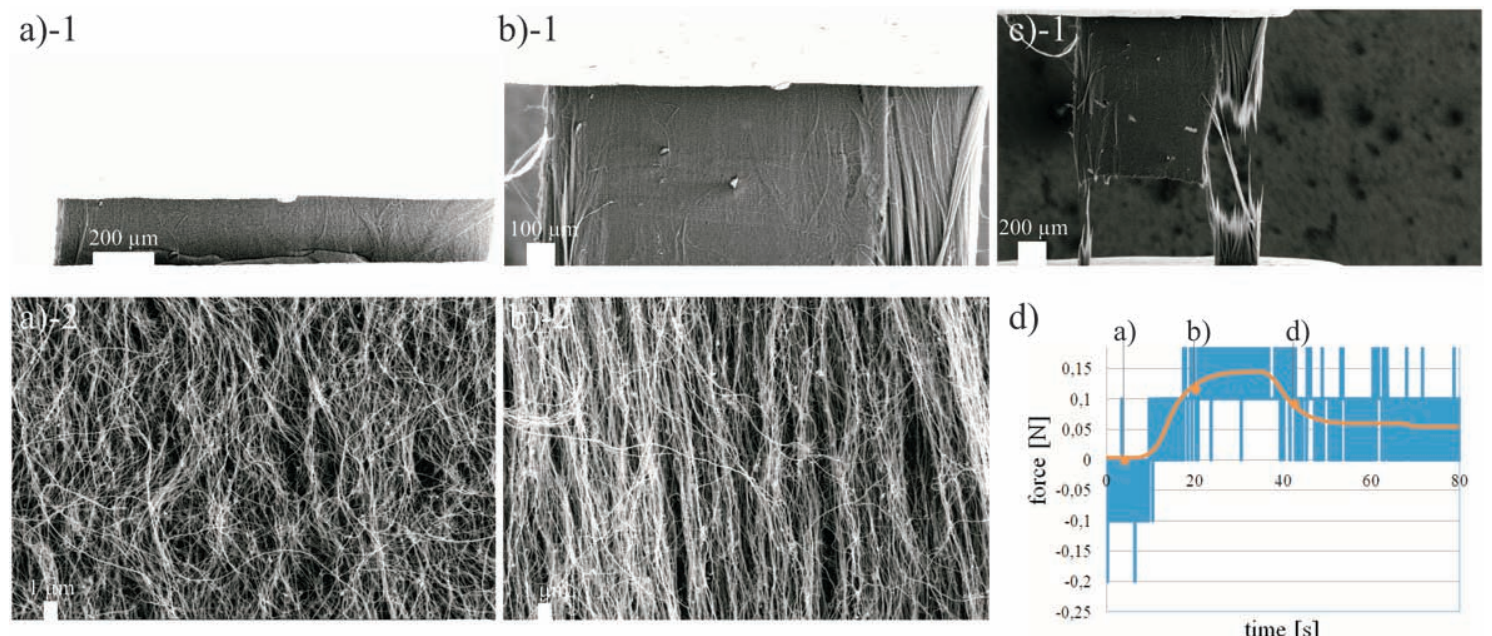

Figure 9. SEM-images of MWCNT-arrays:

a)-1: View on the sample fixed between the mounting at $0 \mu \mathrm{m}$.

b)-1: View on the streched sample at $602 \mu \mathrm{m}$.

c)-1: View on the almost failed sample at $1521 \mu \mathrm{m}$.

a)-2: Detailed view on the curly, starting shape of the MWCNTs.

b)-2: Detailed view on the aligned tubes.

d) Force-time graph of the experiment with the assigned images.

can be seen in Figure9 c)-1. Although the mounting has to be improved the presented test demonstrates the possibility of a further nanotube alignment.

\section{CONCLUSIONS}

The actuated tensile tests revealed a strongly condition- and charging-depentend active behavior of CNT-papers. However, a testing within the elastic range of the paper is a possible way to reduce significantly the standard deviation in order to reveal sensitive effects. The measured effects can be explained using the presented papermodel of interconnected CNT-flakes. An ion diffusion-induced actuation mechanism seems to be the most suitable explanation. In contrast MWCNT-arrays show none of these dependencies. The test set-up and sample alignment suggest a quantum-mechanical effect of the carbon bonds. The alignment of the actuated tensile tested MWCNTs is of special interest as it is an important prerequiste for avoiding secondary effects such as swelling as result of ion-diffusion. That is the reason for the conducted micro tensile test to prove a further nanoscale alignment of the former wavy MWCNT-arrays by a microscale test set-up. In the presented approach the alignment can be obviously documented and the earlier made statements regarding a quantum-mechanical-induced actuation mechanism of MWCNTs-arrays is further supported.

\section{ACKNOWLEDGMENTS}

This work is part of the basic research on future smart materials at the DLR - Institute of Composite Structure and Adaptive Systems. It was supported by the German Research Council (DFG) within the framework of the DFG PAK 355 - 'Basics for CNT-based Actuators' and the German Federal Ministry of Education and Research (BMBF) project 'Aktu_Komp'. Tribute has also to be paid to the colleagues of the Institute of Composite Polymers at the Technical University of Hamburg-Harburg (TUHH), the Institute of Surface and Thin Film Technology at the University of Applied Science Wismar (HSW) as well the Institute of mechanical Process Engineering, department of Interface Chemistry at the University of Clausthal-Zellerfeld for supporting the presented research with their analysis techniques and expertise. 


\section{References}

[1] L., V., Radushkevich, V., M., Lukyanovich, "Clear Images of 50 Nanometer Diameter Tubes made of Carbon," Soviet Journal of Physical Chemistry 62 (1952).

[2] M., Oberlin, M., Endo, "Filamentous Growth of Carbon Through Benzene Decomposition," Journal of Crystal Growth 32, 335-349 (1976).

[3] S. Iijima, "Helical microtubules of graphitic Carbon," Nature 354, 56-58 (1991).

[4] M.-F. Yu, O. Lourie, M. J. Dyer, K. Moloni, T. F. Kelly, R. F. Ruoff, "Strenght an Breaking Mechanism of Multiwalled Carbon Nanotubes Under Tensile Load," Science 287, 637-640 (2000).

[5] M.-F., Yu, B., S., Files, S., Arepalli, et al., "Tensile Loading of ropes of Single Wall Carbon Nanotubes and their Mechanical Properties," Phys. Rev. Lett. bf, 5552-5555 (2000).

[6] U. Vohrer, N. Zschoerper, "Kohlenstoff-Nanoröhren - Phönix aus der Asche," Vakuum in Forschung und Praxis 19(2), 22-30 (2007).

[7] N. Grobert, "Nanotubes - grow or go?," Mater. Today $\mathbf{9}(10)$ (2006).

[8] F. Kreupl, "Electronics: Carbon nanotubes finally deliver," Nature 484, 321-322 (2012).

[9] Z., C., Tu, X., Hu,, "Molecular motor constructed from a double-walled carbon nanotube driven by axially varying voltage," Phys. Rev. B. $72(3), 033404$ (2005).

[10] H., K., Baloch, N. Voskanian, M., Bronsgeest, J., Cumings, "Remote jule heating by a carbon nanotube," Nature Nanotechnology Letter 7, 316-319 (2012).

[11] R. H. Baughman et al., "Carbon nanotube actuators," Science 284, 1340-1344 (1999).

[12] H. P. Monner, S. Mühle, P. Wierach and J. Riemenschneider, "Carbon nanotubes - ein multifunktionaler Leichtbauwerkstoff für die Adaptronik," Adaptronic Congress, 1st-3rd of April, Wolfburg, Germany 2, 1-7 (2003).

[13] P. Wierach, A. Schönecker, "Bauweisen und Anwendungen von Piezokompositen in der Adaptronik," Adaptronik Congress, 31st of May - 01st of June, Göttingen, Germany 4, 1223-1225 (2005).

[14] Bar Cohen, Y., [Actuators as artificical muscles: reality, potential and challenges], SPIE - The International Society for Optical Engineering, Bellingham, Washington, 2 ed. (2004).

[15] T., Mirfakhrai, J. Oh, M., Koslov, E., C., W., Fok, M., Zhang, S., Fang, R., H., Baughman, J., D., W., Madden, "Electrochemical actuation of carbon nanotube yarns," 16, 243-249 (2007).

[16] J. Riemenschneider, H. Temmen and H. P. Monner, "CNT based actuators: Experimental and theoretical investigation of the in-plain strain generation," J. Nanosci. Nanotech. 7(10), 3359-3364 (2007).

[17] A. Minett, J. Fraysse, G. Gang, G.-T. Kim, S. Roth, "Nanotube actuators for nanomechanics," Curr. Appl. Phys. 2, 61-64 (2002).

[18] S. Gupta, M. Hughes, A. H. Windle, J. Robertson, "In situ Raman spectro-electrochemistry study of singlewall carbon nanotubes mat," Diam. Relat. Mater. 13, 1314-1321 (2004).

[19] D. Suppiger, S. Busato, P. Ermanni, M. Motta, A. Windle, "Electromechanical actuation of marcoscopic carbon nanotube structures: mats and sligned ribbons," Phys. Chem. Chem. Phys. 11, 5180-5185 (2009).

[20] P. G. Whitten, G. M. Spinks, G. G. Wallace, "Mechanical properties of carbon nanotube paper in ionic liquid and aqueous electrolytes," Carbon 43, 1891-1896 (2005).

[21] G. Spinks, G. G. Wallace, L. S. Fifield, L. R. Dalton, A. Mazzoldi, D. De Rossi, I. I. Khayrullin, R. H. Baughman, "Pneumatic Carbon Nanotube Actuators," Adv. Mater. 14(23), 1728-32 (2002). 
[22] Y.H. Yun, V. Shanov, Y. Tu, M. J. Schulz, S. Yarmolenko, S. Neralla, J. Sankar, S. Subramaniam, "A Multi-Wall Carbon Nanotube Tower Electrochemical Actuator," Nano Lett. 6(4), 689-693 (2006).

[23] S. Geier, T. Mahrholz, P. Wierach, M. Sinapius, "Carbon nanotubes array actuators," Smart. Mater. Struct. 22(9), 094003 (2013).

[24] U., Vainio, T., I., W., Schnoor, S., K., Vayalil, K., Schulte, M., Müller, E., T., Lilleodden, "Orientation Distribution of Vertcally Aligned Multiwalled Carbon Nanotubes," J. Phys. Chem. C 118, 9507-9513 (2014).

[25] J. Wittenburg, E. Pestel, "Festigkeitslehre, Ein Lehr- und Arbeistbuch," Springer, Berlin Heidelberg New York 3 (2001).

[26] S. M. Geier, T. Mahrholz, P. Wierach, M. Sinapius, "Clarifying the carbon nanotube actuation mechanism - an experimental approach," Proceedings of the ASME 2012 Conference on Smart Materials, Adaptive Structures $\&$ Intelligent Systems SMASIS2012, 19th-21st Sep. 2012, Stone Mountain, Georgia, USA SMASIS2012-8072 (2012).

[27] S. M. Geier, T. Mahrholz, P. Wierach, M. Sinapius, "Carbon nanotube strain measurements via tensile testing," Proceedings of the ASME 2014 Conference on Smart Materials, Adaptive Structures 85 Intelligent Systems SMASIS2014, 8th-10th Sep. 2014, Newport, Rhode Island, USA SMASIS2014-7572 (2014).

[28] E., R., Nightingale, "Phenomenological Theory of Ion Solvation. Effective Radii of Hydrated Ions," J. Phys. Chem. 63(9), 1381-1387 (1959). 